\title{
Forensic Application of Palatal Rugae in Dental Identification
}

\author{
Sartaj Singh Wazir, ${ }^{1}$ Pallok Arora, ${ }^{2}$ Rahul Srivastava, ${ }^{3}$ Sameer Rastogi ${ }^{4}$ \\ 'Department of Oral Medicine \& Radiology, M.B Kedia Dental College, Birganj, Nepal, ${ }^{2,4}$ Department of Oral Medicine \& \\ Radiology, Kalka Dental College, Hospital and Research Centre, Meerut, India, ${ }^{3}$ Department of Oral Medicine \& Radiology, \\ Rama Dental College, Hospital and Research Centre, Kanpur, India.
}

\section{ABSTRACT}

Introduction: The palatal rugae are anatomical folds or wrinkles located on the anterior third of the palate behind the incisive papilla on each side of the palatal raphe. The rugae patterns have been studied for various purposes mainly in the field of anthropology, comparative anatomy, genetics, prosthodontics, orthodontics and forensic odontology. This study is aimed to determine the difference in number and pattern of palatal rugae in males and females.

Methods: This is a prospective study in which maxillary impressions of 50 volunteers, 25 males and 25 females were taken and the casts prepared. The boundaries of the rugae were marked with a HB pencil and were observed with a magnifying glass following the classification used by Kapali, et al, (1997). Association between rugae number and sex and rugae shape and sex were tested using two sample unpaired $t$ test.

Results: The study revealed significant difference in the pattern of converge rugae which were found to be higher in females than males, however, no statistical significant difference was found in the number of rugae between the two sexes.

Conclusions: The rugae pattern can be an additional method of differentiation between the males and females in conjunction with the other methods used in forensic sciences.

Keywords: forensic science; human identification; palatal rugae.

\section{INTRODUCTION}

To establish a person's identity, fingerprints, DNA and dental comparisons are probably the most commonly used techniques. ${ }^{1}$ Over the last few decades, the use of teeth for post mortem identification has gained a lot of importance, however, in the edentulous patients, it is not possible. ${ }^{2}$ The palatal rugaeat this juncture can be used successfully in human identification., ${ }^{1,3-5}$

The use of palatal rugae for personal identification was first suggested by Allen in $1889 .{ }^{6}$ The rugae possesses the features of an ideal forensic identification parameter i.e. they are unique to an individual, can resist postmortem insults and remain stable after the completion of the growth. It can be effectively applied in cases of completely burnt bodies; that have undergone severe decomposition, in completely edentulous victim's etcprovided an ante mortem record exists. ${ }^{7}$ 
This study was conducted to determine the role of palatal rugae in forensic identification.

\section{METHODS}

This is a prospective study conducted at Rama Dental College, Hospital and Research Centre, Kanpur, from September 2009 to December 2009. Ethical approval was obtained from the Ethical Committee of the Institutional Review Board and verbal consent was taken from all the patients.

Healthy individuals with an age ranging from $12-30$ years who had come for the treatment of various dental problems were included in the study. Patients with any congenital palatal deformities, high palatal vaults and systemic diseases affecting the oro-facial tissues were excluded from the study.

The total sample consisted of 50 casts 25 of males and 25 of females of North Central India. An irreversible hydrocolloid was used as an impression material in an appropriate perforated metal tray for the upper dental arch for all subjects. The impressions were then poured with dental stone and the bases were made with plaster of Paris. All casts were free of air bubbles or voids. The rugae were delineated using a black HB pencil under adequate light and magnification and recorded according to the classification given by Kapali, et al, (Figure 1). ${ }^{8}$ Rugae were categorized as straight, wavy, curved and circular. In addition, if rugae had two arms, it was categorised as 'unification.' When two rugae originate away from the centre and unite towards it, the unification is categorized as converge whereas diverge ones are those rugae which originate from the centre and diverge away from it.

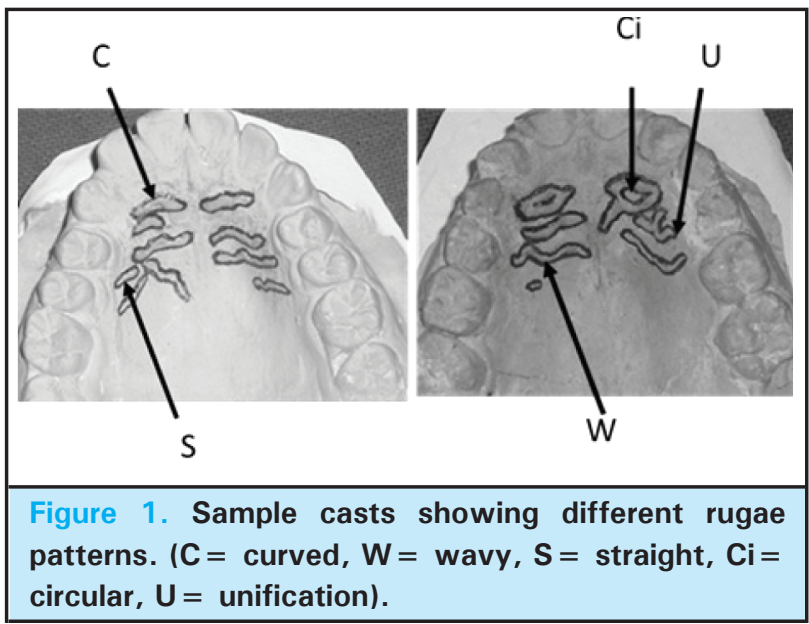

All the casts were studied for the number and pattern of rugae. In this study, the fragmented types of rugae of a size less than $5 \mathrm{~mm}$ were ignored, when the mean value of the total number of rugae was calculated.

The association between rugae number and sex, and rugae shape and sex were analyzed using statistical package for software analysis (SPSS) software using two sample unpaired t test. A $p$ value of $<0.05$ was taken to be significant.

\section{RESULTS}

There was a significant difference in the converge type of rugae which was found to be higher among females $(7.36 \%)$ than males $(2.02 \%)(p=.0234)$. There was also some difference in circular type which was higher in males $(3.03 \%)$ than females $(1.05 \%)$ but was not statistically significant $(p=0.23$ ) (Table 1). However, there was no significant difference in the total number of rugae in males and females ( $p=0.5013$ ) (Table 2)

\begin{tabular}{|c|c|c|c|c|c|c|}
\hline Type & Sex & No. & Mean & S.D & SEM & $p$-value \\
\hline \multirow{2}{*}{ Curved } & M & 25 & 3.28 & 1.40 & 0.28 & \multirow{2}{*}{0.5838} \\
\hline & $\mathrm{F}$ & 25 & 308 & 115 & 023 & \\
\hline \multirow{3}{*}{ Wavy } & $M$ & 25 & 2.76 & 1.42 & 0.28 & \multirow{3}{*}{0.2856} \\
\hline & & & & & & \\
\hline & $F$ & 25 & 2.36 & 1.19 & 0.24 & \\
\hline \multirow{3}{*}{ Straight } & $M$ & 25 & 1.28 & 0.89 & 0.18 & \multirow{3}{*}{0.6702} \\
\hline & & & & & & \\
\hline & $F$ & 25 & 1.40 & 1.08 & 0.22 & \\
\hline \multirow{3}{*}{ Circular } & $M$ & 25 & 0.24 & 0.60 & 0.12 & \multirow{3}{*}{0.2302} \\
\hline & & & & & & \\
\hline & $\mathrm{F}$ & 25 & 0.08 & 0.28 & 0.06 & \\
\hline \multirow{3}{*}{ Converge } & $M$ & 25 & 0.16 & 0.47 & 0.09 & \multirow{3}{*}{$0.0234 *$} \\
\hline & & & & & & \\
\hline & $F$ & 25 & 0.56 & 0.71 & 0.14 & \\
\hline \multirow{3}{*}{ Diverge } & $M$ & 25 & 0.20 & 0.41 & 0.08 & \multirow{3}{*}{0.4507} \\
\hline & & & & & & \\
\hline & $\mathrm{F}$ & 25 & 0.12 & 0.33 & 0.07 & \\
\hline
\end{tabular}

Two sample unpaired t-test was performed. $M=$ Male

$\mathrm{F}=$ Female

* indicates significant difference 


\begin{tabular}{|l|cccccc|}
\hline Table 2. Statistical description of total number of \\
rugae in males and females. \\
\hline Sex & $\begin{array}{c}\text { Total } \\
\text { Number } \\
\text { of } \\
\text { Sample }\end{array}$ & $\begin{array}{c}\text { Total } \\
\text { Number Rugae }\end{array}$ & Mean & S.D & SEM & p-value \\
Males & 25 & 198 & 7.92 & 2.18 & 0.44 & \\
Females & 25 & 190 & 7.60 & 0.91 & 0.18 & $0.5013^{*}$ \\
\hline
\end{tabular}

* indicates no significant difference.

The distribution of different types of rugaein both the sexes as a whole were curved $(40.98 \%)$ followed by wavy (32.99\%), straight $(17.27 \%)$, converge $(04.64 \%)$, circular (02.06 \%) and diverge $(02.06 \%)$ (Figure 2).Sex wise also, the curved and the wavy types were the most frequent rugae patterns with a percentage of $41.41 \%$ and $34.85 \%$ in males and $40.53 \%$ and $31.05 \%$ in females respectively.

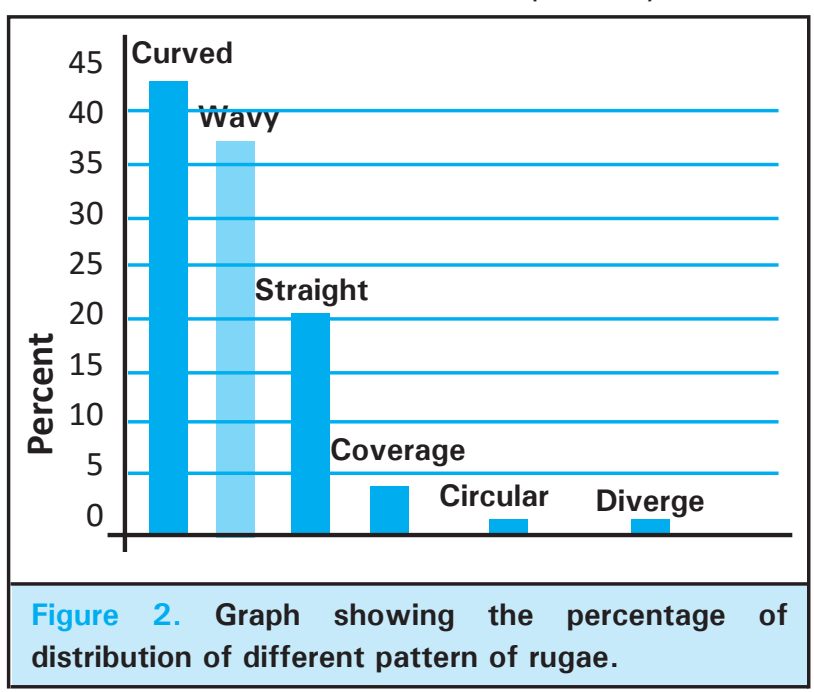

\section{DISCUSSION}

Many attempts have been made in order to find out the safe means for human identification that include anthropometry, fingerprints, gender determination, age estimation, weighing, and identification by specific characteristics, and blood group differentiation. ${ }^{8}$ Investigation of the mucosal tissues other than the teeth and the jaws is also important, since these structures provide interesting data for identification, such as the hard palate, by means of investigation of palatal rugae pattern (rugoscopy). ${ }^{9}$ Summitt J.B, et al, (1988) has also concluded that the palatal rugae pattern is sufficiently characteristic to discriminate between individuals. ${ }^{10}$

Previous studies that described population variations in rugae have usually employed classifications based on rugae shape, unification and length, with some claiming this approach as the most accepted. ${ }^{2,3,8,11}$ PreetiNayak, et al (2007)has mentioned that Thomas and Kotze, (1983) have highlighted the difficultiesin observing, classifying and interpreting the limitless and minute variations in palatal rugae, and emphasized the necessity for standardizing the procedure. ${ }^{3}$ However, theyconcede that a universally acceptable classification is improbable and suggest that "a single operator alone (eliminating inter-observer error), using his own classification can very successfully apply it in a comparative project and that the results of the comparisons and not the classification itself are important." It has been suggested that a classification system that is simple and reliable be used in rugae studies. ${ }^{8}$ Number of classifications have been put forward by various authors to classify the palatal rugae..$^{1,7,8}$ These include:

\section{Carrea Classification:}

Type I Posterior-anterior directed rugae.

Type II Rugae perpendicular to the raphe.

Type III Anterior-posterior directed rugae.

Type IV Rugae directed in several directions.

\section{II. da Silva Classification:}

$\begin{array}{ll}\text { 1. } & \text { Line } \\ \text { 2. } & \text { Curve } \\ \text { 3. } & \text { Angle } \\ \text { 4. } & \text { Circle } \\ \text { 5. } & \text { Wavy } \\ \text { 6. } & \text { Point }\end{array}$

III. Trobo Palatal Classification:

$\begin{array}{ll}\text { Type A } & \text { Point } \\ \text { Type B } & \text { Line } \\ \text { Type C } & \text { Curve } \\ \text { Type D } & \text { Angle } \\ \text { Type E } & \text { Sinuous } \\ \text { Type F } & \text { Circle }\end{array}$

IV. Martins dos Santos classification:

Based on the form and position of each palatal rugae, this classification indicates and characterizes the following:

i. One initial rugae: The most anterior one on the right side is represented by a capital letter.

ii. Several complimentary rugae: The other right rugae are represented by numbers.

iii. One sub initial rugae: The most anterior one on the left side is represented by a capital letter.

iv. Several subcomplimentary rugae: The other left rugae are represented by numbers.

V. Thomas et al Classification, (1983):

This classification is based on the number, length, shape and unification of the rugae. (Figure 3) 


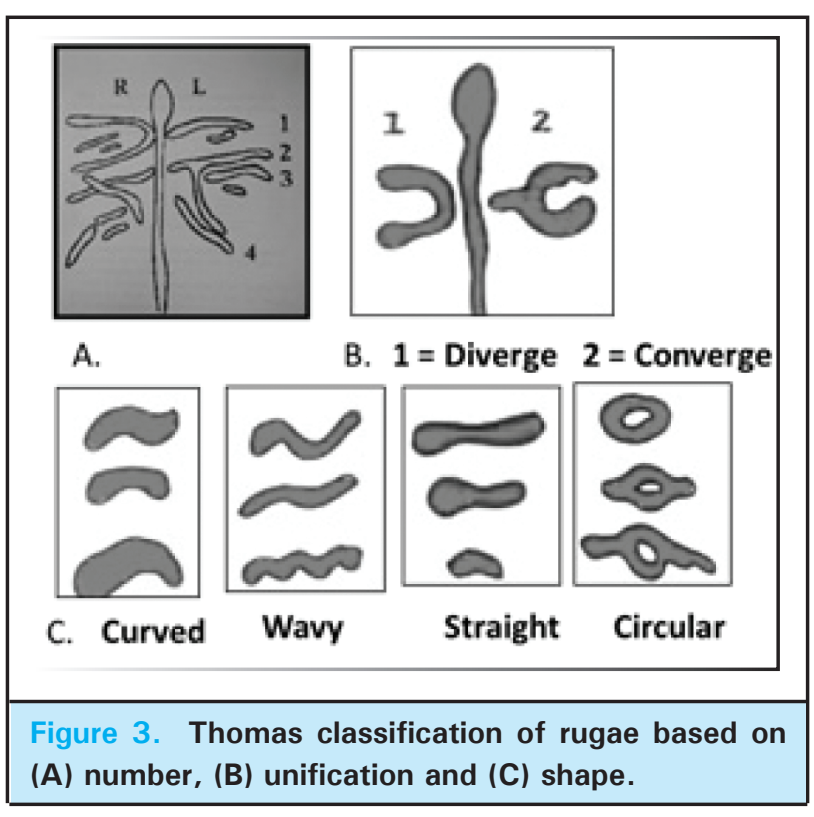

i. Based On Number

$$
1,2,3,4
$$

ii. Based on length

Primary rugae 5 to $10 \mathrm{~mm}$

Secondary rugae 3 to $5 \mathrm{~mm}$

Fragmentary rugae less than $3 \mathrm{~mm}$

iii. Based on unification

Converge

Diverge

iv. Based on shape

Curved

Wavy

Straight

Circular

The classification of SunitaKapali, et al, (1997) was followed for recording the rugae patterns as was done in the study by Faisal M Fahmi, et al, (2001) and PreethiNayak, et al, (2007)., ${ }^{2,3}$ The rugae were categorized as straight, wavy, curved, circular and as unifications (converge and diverge).

Many studies have been conducted on the rugae patterns in Blacks, Australian Caucasians and Aborigines, Japanese, Tibetans and Southern and
Western India. 2,3,6,8,11 However, no study have been done in the North Central part of India. This study did not show any significant difference in the number of rugae between the males and the females $(p=0.5013)$ like the study conducted by Faisal M Fahmi, et al, (2001). ${ }^{2}$ These results however donot conform with the results presented by Dohke and Osato, (1994) who have mentioned that among the Japanese, the females had fewer rugae than the Japanese males. ${ }^{12}$ This finding may indicate that there is some racial and sex difference. In this study, the most common type of rugae observed in both the sexes were curved and wavy which is consistent with the findings in Australian Aborigines and Caucasians where wavy and curved were the most prevalent types while straight, curved and unifications were less common. ${ }^{8}$ But, in our study, the straight rugae carried a good percentage as was found in the study by PreethiNayak, et al (2007). ${ }^{3}$ In our study, the circular rugae, of course less in number were recorded similar to the study conducted by Faisal M. Hashmi, et al, (2001) and in contrast to the study conducted by PreethiNayak, et al, (2007)where circular rugae were totally absent. ${ }^{2,3}$

However, in this study, statistically significant difference was seen in the converge type of the rugae which was found to be higher in the females than the males $(p=$ $0.0234)$. Similarly, in the study conducted by Faisal M Fashmi, (2001) the females showed a predilection for the converge type, but in addition, they have also found the circular type to be more prevalent in the males. ${ }^{2}$ In our study, of course, there was a difference in the circular types among the males and the females but was not statistically significant may be because of smaller sample size. These two differences could be the factors for identification together with other methods of identification. Since, the sample used in this study was small, further studies with a larger sample are justified in order to substantiate the usefulness of human palatal rugae for the human identification.

\section{CONCLUSIONS}

Significant differences were found in the converge type which was found to be higher in females. There was no significant difference in total number of rugae between males and females. The rugae pattern may be a useful additional method for post mortem identification provided an ante mortem record exists.

\section{REFERENCES}

1. Caldas IM, Magalhaes T, Afonso A. Establishing identity using cheiloscopy and palatoscopy. Forensic Sci Int. 2007;165:1-9.
2. Faisal MF, Saleh MAS, Yousef FT. Rugae pattern in a Saudi population sample of males and females. Saudi Dental Journal. 2001;13(2):92-5. 
3. Nayak P, Acharya AB, Padmini AT, KaveriH. Differences in the palatal rugae shape in two populations of India. Arch Oral Biol. 2007;52:977-82.

4. Stavrianos C, Stavrianou I, Dietrich EM, Kafas P. Methods for human identification in Forensic Dentistry: A Review. Internet Journal of Forensic Science. 2009;4(1):5.

5. Segelnick SL, LeonardG. Forensic application of palatal rugae in dental identification. Forensic Examiner The Spring. 2005;14(1):22-6.

6. Maki O, Naoki N, Takashi C, Masayuki F, YojiM, Naofumi $\mathrm{Y}$. Indication and limitations of using palatal rugae for personal identification in edentulous cases. Forensic Sci Int. 2008;176:178-82.
7. Rugae pattern in Indian population. Indian Dent AssecTimes. 2008 May 9. http//www.ida.org.com.

8. Kapali S, Townsend G, Richards L, Parish T. Palatal rugae patterns in Australian Aborigines and Caucasians. Aust Dent J. 1997;42(2):129-33.

9. Ismar EMF, Sílvia HCSP, Arsenio SP, Suzana PMC. Palatal rugaepatterns as bioindicatorsof identification in Forensic Dentistry.RFO. 2009;14(3):227-33.

10. English WR, Robison SF, Summitt JB, Oesterle LJ, Brannon RB, Robison SF, et al. Individuality of human palatal rugae. JForensicSci. 1988;33(3):718-26.

11. Shetty SK, Kalia S, Patil K, Mahima VG. Palatal rugae pattern in Mysorean and Tibetan populations. Indian J Dent Res. 2005;16(2):51-5.

12. Dohke M, Osato S. Morphological study of the palatal rugae in Japanese Bilateral differences in the regressive evaluation of the palatal rugae. Jap J Oral Biol. 1994;36:125-40. 